Article

\title{
How Culture and Sociopolitical Tensions Might Influence People's Acceptance of COVID-19 Control Measures That Use Individual-Level Georeferenced Data
}

\author{
Jianwei Huang ${ }^{1}\left(\mathbb{D}\right.$, Mei-Po Kwan ${ }^{1,2, *(D)}$ and Junghwan $\operatorname{Kim}^{3}(\mathbb{D})$ \\ 1 Institute of Space and Earth Information Science, The Chinese University of Hong Kong, Shatin, Hong Kong; \\ jianwei.huang@link.cuhk.edu.hk \\ 2 Department of Geography and Resource Management, The Chinese University of Hong Kong, \\ Shatin, Hong Kong \\ 3 Center for Geographic Analysis, Institute for Quantitative Social Science, Harvard University, \\ Cambridge, MA 02138, USA; junghwan_kim@fas.harvard.edu \\ * Correspondence: mpkwan@cuhk.edu.hk
}

check for updates

Citation: Huang, J.; Kwan, M.-P.; Kim, J. How Culture and Sociopolitical Tensions Might Influence People's Acceptance of COVID-19 Control Measures That Use Individual-Level Georeferenced Data. ISPRS Int. J. Geo-Inf. 2021, 10, 490. https://doi.org/10.3390/ ijgi10070490

Academic Editor: Wolfgang Kainz

Received: 9 June 2021

Accepted: 16 July 2021

Published: 20 July 2021

Publisher's Note: MDPI stays neutral with regard to jurisdictional claims in published maps and institutional affiliations.

Copyright: (c) 2021 by the authors. Licensee MDPI, Basel, Switzerland. This article is an open access article distributed under the terms and conditions of the Creative Commons Attribution (CC BY) license (https:// creativecommons.org/licenses/by/ $4.0 /)$
Abstract: This study extends an earlier study in the United States and South Korea on people's privacy concerns for and acceptance of COVID-19 control measures that use individual-level georeferenced data (IGD). Using a new dataset collected via an online survey in Hong Kong, we first examine the influence of culture and recent sociopolitical tensions on people's privacy concerns for and acceptance of three types of COVID-19 control measures that use IGD: contact tracing, self-quarantine monitoring, and location disclosure. We then compare Hong Kong people's views with the views of people in the United States and South Korea using the pooled data of the three study areas. The results indicate that, when compared to people in the United States and South Korea, people in Hong Kong have a lower acceptance rate for digital contact tracing and higher acceptance rates for self-quarantine monitoring using e-wristbands and location disclosure. Further, there is geographic heterogeneity in the age and gender differences in privacy concerns, perceived social benefits, and acceptance of COVID-19 control measures: young people (age < 24) and women in Hong Kong and South Korea have greater privacy concerns than men. Further, age and gender differences in privacy concerns, perceived social benefits, and acceptance of COVID-19 control measures in Hong Kong and South Korea are larger than those in the United States, and people in Hong Kong have the largest age and gender differences in privacy concerns, perceived social benefits, and acceptance of COVID-19 measures among the three study areas.

Keywords: COVID-19; privacy; geoprivacy; contact tracing; self-quarantine; age and gender difference

\section{Introduction}

As the COVID-19 pandemic became a major global health crisis, many COVID-19 control measures that use individual-level georeferenced data (e.g., the locations of people's residences and activities) have been used in many countries around the world. These measures include contact tracing, self-quarantine monitoring, and location disclosure [1-5]. Contact tracing identifies the persons who had been in close contact with infected persons and the places or venues (with location information) infected persons recently visited. When implemented via various digital technologies, digital contact tracing (e.g., e-wristbands, mobile phone GPS tracking, and social media location) enables governments to obtain individual-level georeferenced data (IGD) and develop response measures more rapidly than when using conventional contact tracing methods [6-10]. Self-quarantine monitoring measures check on people required to self-quarantine to see if they are adhering to the self-quarantine requirements (i.e., staying where they are supposed to be). Location disclosure involves disclosing (and often publicizing) the places or venues visited by infected persons so that other people may avoid visiting these locations and venues $[6,11-14]$. The 
implementation of effective self-quarantine monitoring measures and location disclosure also need IGD.

However, because of the precise locations they contain, georeferenced data have considerable potential for disclosing people's identity through a process known as spatial reverse engineering (e.g., by linking high-resolution geospatial data with other data such as census or survey data to discover the identity of specific individuals) [15-19]. The effectiveness and potential benefits of using individual-level georeferenced data (IGD) in COVID-19 control measures may thus be undermined by people's geoprivacy concerns for and acceptance of these measures, where geoprivacy is the privacy (the right to protect and prevent such information from being disclosed to the public) of people's residential and activity locations (e.g., workplace) [20].

Social scientists have highlighted certain social and behavioral factors that significantly affect people's acceptance and the effectiveness of COVID-19 control measures. For instance, Bavel et al. [21] suggested that people's behavior is influenced by social norms. Further, some differences in people's responses to the COVID-19 pandemic may be understood in terms of cultural differences. As Bavel et al. [21] argued, Western European and North American cultures that endorse individualism tend to value individual independence and the expressions of the self more than Asian cultures, and this may lead to a higher likelihood of interpersonal transmission of the coronavirus. On the other hand, the priority given to obligations to the larger society (collectivism) by people in Asian countries may motivate them to be more committed to adhering to certain COVID-19 control measures (e.g., wearing masks and social distancing) at the cost of some individual freedom when compared with people in Western European and North American countries. Note that, in addition to cultural factors, the collective experience of a recent infectious disease outbreak, like the Severe Acute Respiratory Syndrome (SARS) in 2003 in Hong Kong, may also motivate people to act more cooperatively (i.e., in a collectivist-like manner) and be more committed to adhering to certain COVID-19 control measures (more discussion on this later in this article).

Habersaat et al. [22] (p. 679) have also put forward similar ideas concerning the influence of behavioral and cultural factors on the effectiveness of COVID-19 control measures (e.g., the need to balance individual rights with the social good). They argued that people's "willingness to act for the benefit of society is subject to cultural differences and is more prominent in collectivist countries than in individualistic countries, where maximizing individual benefit is prioritized". These cultural differences may influence people's acceptance of different COVID-19 control measures [22].

Along this line, studies using individual-level data have examined people's perception of COVID-19 risk, related control measures, and the effectiveness of government policies in different countries (e.g., [23]). For example, de Bruin and Bennett [24] found that people in the United States who perceive greater COVID-19 risks are more likely to adopt protective behaviors. An international study by Dryhurst et al. [25] assessed people's attitudes towards the risk of COVID-19 and government reactions in ten different countries. The study also investigated whether different cultural norms and different experiences of the pandemic have any effects on people's feelings of trust and perceptions of risk across countries. It found that individualistic worldviews, personal experience with COVID-19, and trust in government are significant determinants of people's risk perception (although there is substantial variability across cultures). In a study of 57 countries at the national level, Gelfand et al. [26] found that countries with efficient governments and tight cultures are most successful in controlling the spread of the COVID-19 pandemic and the associated mortality (where cultural tightness is defined as the strength of norms in a country and the tolerance for people who violate norms). Further, as New Zealand's success in controlling the COVID-19 pandemic indicates, a highly effective government can still flatten the curve even when a country does not have a collectivist orientation or a tight culture.

While these studies have enhanced our understanding of people's COVID-19 risk perception and the influence of cultural and institutional factors (e.g., individualistic 
orientation, the strengths of and adherence to social norms, and trust in government) on the overall effectiveness of COVID-19 control measures in different countries, few studies to date have examined how people's privacy concerns influence their acceptance of COVID19 control measures that use IGD (which in turn may have a significant influence on the potential effectiveness of these measures). However, because some Asia countries (e.g., China, Singapore, and South Korea) have achieved impressive performance in "flattening the curve", partly by deploying digital contact tracing in the early phase of the COVID19 pandemic $[27,28]$, people who live in these countries do seem to be more willing to embrace digital contact tracing that provides their IGD to governments despite their privacy concerns [29-32]. Note that people's personal location information gathered via digital contact tracing using various devices (e.g., mobile phone applications) is automatically sent to public health agencies, which may eventually lead to some possibilities for geoprivacy violations $[33,34]$. The collectivist orientation of the people in these Asian countries, however, may have made COVID-19 measures that use IGD more acceptable than among people in Western countries, despite privacy concerns [35,36].

A recent study by Kim and Kwan [33] provides important insights into these issues. The study examined people's privacy concerns, perceived social benefits, and acceptance of COVID-19 control measures that use IGD in the United States and South Korea. It found that people have higher privacy concerns for measures that use more personal information and that people's perceived social benefits of various measures are low when their privacy concerns are high. It also observed that people in South Korea have higher acceptance for the control measures than people in the United States and that South Koreans (when compared with people in the Unites States) and people with a stronger collectivist orientation have higher acceptance for the control measures. These findings advance our understanding of the important role of culture in influencing people's acceptance of different COVID-19 control measures.

In this paper, we extend this earlier study on the United States and South Korea by including Hong Kong as a third study area, which arguably lies between the United States and South Korea on the individualist-collectivist spectrum (partly owing to its history as a British colony from 1841 to 1997) [37,38]. Specifically, using a new online survey dataset collected in Hong Kong, we assess Hong Kong people's privacy concerns, perceived social benefits, and acceptance for ten COVID-19 control measures. We also examine the differences in these three aspects between people in Hong Kong and those in the United States and South Korea by pooling together our new dataset with the online survey dataset collected by Kim and Kwan [33] (however, note that in the United States, accepting public health advice might have led to lower perceived social benefits and acceptance of COVID-10 control measure owing to the ill-conceived public health advice of the government in 2020).

In this paper, we will proceed by first investigating Hong Kong people's views on COVID-19 control measures. Then, we compare how people's views on COVID-19 control measures differ among Hong Kong, the United States, and South Korea. Finally, we examine and compare the associations between participants' sociodemographic characteristics, collectivist orientation, and the residing areas on one hand, and participants' privacy concerns, perceived social benefits, and acceptance of COVID-19 control measures on the other between the three study areas (i.e., Hong Kong, the United States, and South Korea).

\section{Study Areas, Data, and Methods}

\subsection{Study Areas}

This study extends an earlier study on the United States and South Korea by including Hong Kong as a third study area. Hong Kong is a well-known global city in Asia with a population of about 7.5 million in 2020. It has been quite successful in controlling the spread of COVID-19. As of early June 2021, there have been 11,852 confirmed COVID-19 cases and 210 deaths in Hong Kong. The main COVID-19 control measures in Hong Kong are border control (e.g., a 14-day self-quarantine for in-bound travelers), social distancing (e.g., closure 
of schools, suspension of public services), contact tracing (e.g., in-depth interview), and location disclosure (e.g., buildings and venues visited in the 14 days before infection was confirmed) [39]. These measures were successful in mitigating the COVID-19 pandemic in Hong Kong. Meanwhile, the spread of the pandemic in the United States finally started to slow down under the vaccination program after a year of COVID-19 waves: the daily new confirmed cases declined from 200,000 to around 10,000 after the implementation of the COVID-19 vaccination program from January 2021 to June 2021 [40]. However, South Korea has recently (i.e., July 2021) experienced its worst COVID-19 outbreaks since the pandemic began owing to the spread of the Delta variant; as of 2 July 2021, South Korea witnessed the highest number of daily confirmed cases (i.e., 800) in nearly six months [41].

Note that Hong Kong people share a similar collective experience of a recent infectious disease outbreak with people in South Korea. In 2003, Hong Kong experienced an outbreak of the Severe Acute Respiratory Syndrome (SARS), while South Korea experienced a Middle East Respiratory Syndrome (MERS) outbreak in 2015. The painful lessons of these serious outbreaks might influence people's perceived social benefits and acceptance of COVID-19 control measures [42]. Further, note that trust in the government for a large number of people in Hong Kong has recently declined to a low level as a result of waves of protests from March 2019 to January 2020 against the attempt of the Hong Kong Government to introduce the Fugitive Offenders Amendment Bill (or the Extradition Bill) [43]. People's low trust in the Hong Kong Government might lead to low acceptance of COVID-19 control measures, as sociopolitical tensions erupted in the form of violent mass street protests. It is thus especially important to investigate Hong Kong people's privacy concerns, perceived social benefits, and acceptance of COVID-19 control measures that use individual-level georeferenced data (IGD), which could provide significant insights into how people might behave and react to government COVID-19 control measures under great sociopolitical tensions.

\subsection{Data Collection}

The data for this research were collected through online surveys via Qualtrics in the United States, South Korea, and Hong Kong using the languages of the respective study areas. The survey questionnaire was originally designed for and implemented in the United States and South Korean surveys in which participants were recruited via Twitter and Facebook from 25 June to 10 July 2020 [33]. Adults of at least 18 years old who had physically stayed in the study areas for any period during the study areas' COVID-19 pandemic were eligible to participate in the survey. A total of 306 participants (188 U.S. participants and 118 South Korean participants) returned complete surveys. The survey questionnaire consisted of two parts: Part 1 solicited participants' views on COVID-19 control measures that use individual-level georeferenced data (IGD), and Part 2 collected the sociodemographic characteristics of survey participants. The survey questionnaire will be described in greater detail in Section 2.3 below.

The Hong Kong survey was conducted from 14 August to 14 September 2020. Participants were recruited through WhatsApp, WeChat, and Twitter. A total of 149 participants returned complete surveys. Thus, in the end, we obtained a total of 455 complete surveys from participants from all three study areas. Note that, although the COVID-19 situations in the United States, South Korea, and Hong Kong were different during the survey periods (Figure 1), all of them had experienced the pandemic for 6 or more months, and at least one significant peak in confirmed COVID-19 cases had happened by the time of the surveys. The study protocol and survey questionnaire were reviewed and approved by the Institutional Review Boards (IRBs) of the authors' universities.

Table 1 presents the participants' sociodemographic attributes in the three study areas. Note that the percentages of female participants in the United States (70\%) and Hong Kong (66\%) surveys are much higher than those in the reference populations in the respective study areas, while the percentage of female participants in the South Korea survey (42\%) is lower than that in the reference population group in the country. Further, note that all 
samples from the three study areas have higher percentages of young and highly educated people when compared with the reference populations of the respective study areas. This is an often encountered sampling bias owing to the recruitment of survey participants via online social media platforms (i.e., Twitter, Facebook, WhatsApp, and WeChat) [33,44]. It is thus important to bear in mind such a sampling bias in our datasets and be cautious when interpreting the results or inferring the situations of other areas.

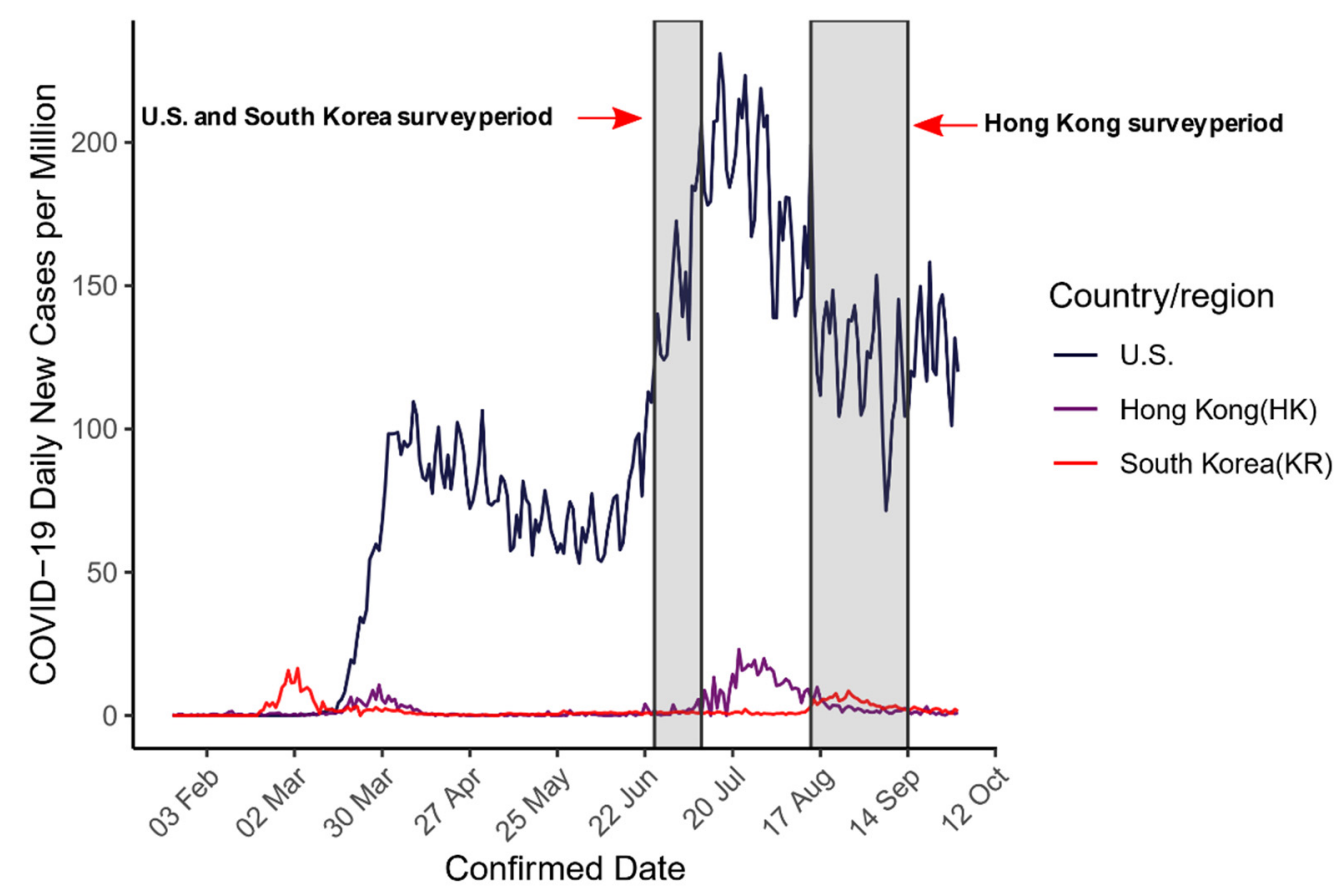

Figure 1. New COVID-19 daily cases per million people in the United States, South Korea, and Hong Kong since January 2020.

Table 1. Sociodemographic characteristics of Hong Kong $(n=149)$, the United States $(n=188)$, and South Korea $(n=118)$ survey participants, and comparison with those of the national/urban populations.

\begin{tabular}{|c|c|c|c|c|c|c|c|}
\hline & & \multicolumn{2}{|c|}{ Hong Kong } & \multicolumn{2}{|c|}{ U.S. } & \multicolumn{2}{|c|}{ South Korea } \\
\hline & & $\begin{array}{c}\text { Sample } \\
(n=149)\end{array}$ & $\begin{array}{c}\text { Urban } \\
\text { Population }\end{array}$ & $\begin{array}{l}\text { Sample } \\
(n=188)\end{array}$ & $\begin{array}{c}\text { National } \\
\text { Population }\end{array}$ & $\begin{array}{l}\text { Sample } \\
(n=118)\end{array}$ & $\begin{array}{c}\text { National } \\
\text { Population }\end{array}$ \\
\hline Gender & Female & $66 \%$ & $55 \%$ & $70 \%$ & $51 \%$ & $42 \%$ & $50 \%$ \\
\hline \multirow{3}{*}{ Age } & $18-24$ & $28 \%$ & $10 \%$ & $26 \%$ & $12 \%$ & $30 \%$ & $14 \%$ \\
\hline & $25-44$ & $52 \%$ & $33 \%$ & $57 \%$ & $34 \%$ & $49 \%$ & $33 \%$ \\
\hline & $45+$ & $19 \%$ & $57 \%$ & $17 \%$ & $53 \%$ & $19 \%$ & $53 \%$ \\
\hline Race & White alone & $\mathrm{N} / \mathrm{A}^{4}$ & $\mathrm{~N} / \mathrm{A}^{4}$ & $55 \%$ & $74 \%$ & N/A 4 & N/A 4 \\
\hline $\begin{array}{l}\text { Higher } \\
\text { Education }\end{array}$ & & $75 \%$ & $33 \%{ }^{5}$ & $88 \%$ & $32 \%^{5}$ & $73 \%$ & $33 \%{ }^{5}$ \\
\hline Student & & $24 \%$ & $\mathrm{~N} / \mathrm{A}$ & $31 \%$ & $\mathrm{~N} / \mathrm{A}$ & $41 \%$ & $\mathrm{~N} / \mathrm{A}$ \\
\hline
\end{tabular}

Notes: ${ }^{1} 2020$ Statistics Hong Kong data (15+ years old). ${ }^{2}$ ACS 2018 five-year estimate data (18+ years old). ${ }^{3}$ 2015/2018 Statistics Korea data (15+ years old). ${ }^{4}$ No race data. ${ }^{5}$ Bachelor's degree or higher (Hong Kong: $15+$ years old; United States: $25+$ years old; South Korea: $20+$ years old).

\subsection{Survey Questionnaire}

The survey questionnaire, which was originally developed for and implemented by Kim and Kwan [33] in the U.S. and South Korea surveys, has two sections. Section 1 of the questionnaire collected data on participants' perceptions of ten COVID-19 control measures that use individual-level georeferenced data (IGD). Section 2 collected data 
about participants' sociodemographic characteristics, individualist-collectivist orientation, and perception of the COVID-19 situation in the study areas. These two sections of the questionnaire are described in greater detail as follows.

Section 1 of the survey questionnaire collected data on participants' views of ten COVID-19 control measures that use IGD. These ten measures belong to three broad types (see Table 2 for details): (1) contact tracing (measures M1-M4); (2) self-quarantine monitoring (measures M5-M8); and (3) public disclosure of the locations of the major activities and personal demographic information (e.g., age and gender) of infected persons (measures M9-M10). Note that, in South Korea, most of these methods (except M4 and M8) have been implemented. In the United States and Hong Kong, however, only a few methods (M1 for the United States, and M1, M7, and M9 for Hong Kong) have been used widely.

Table 2. A detailed description of the ten COVID-19 control measures in the survey.

\begin{tabular}{|c|c|c|c|c|c|}
\hline \multirow[b]{2}{*}{ Method } & \multirow[b]{2}{*}{ Type } & \multirow[b]{2}{*}{ Description } & \multicolumn{3}{|c|}{ Execution } \\
\hline & & & $\begin{array}{l}\text { Hong } \\
\text { Kong }\end{array}$ & U.S. & $\begin{array}{l}\text { South } \\
\text { Korea }\end{array}$ \\
\hline M1 & \multirow{4}{*}{$\begin{array}{l}\text { Contact } \\
\text { tracing }\end{array}$} & $\begin{array}{l}\text { Obtaining location information by conducting conventional } \\
\text { interviews }\end{array}$ & $\mathrm{O}$ & $\mathrm{O}$ & $\mathrm{O}$ \\
\hline $\mathrm{M} 2$ * & & $\begin{array}{l}\text { Obtaining location information from patients' mobile phones } \\
\text { (e.g., GPS trajectories) }\end{array}$ & $x$ & $\Delta$ & $\mathrm{O}$ \\
\hline M3 * & & $\begin{array}{l}\text { Obtaining location information from patients' credit card } \\
\text { history }\end{array}$ & $x$ & $x$ & $\mathrm{O}$ \\
\hline $\mathrm{M} 4$ * & & Bluetooth-based proximity tracing method & $x$ & $\Delta$ & $x$ \\
\hline M5 & \multirow{4}{*}{$\begin{array}{l}\text { Self-Quarantine } \\
\text { Monitoring }\end{array}$} & $\begin{array}{l}\text { Monitoring people's self-quarantine by calling them at random } \\
\text { times of day }\end{array}$ & $\mathrm{O}$ & $\Delta$ & $\mathrm{O}$ \\
\hline M6 * & & $\begin{array}{l}\text { Monitoring people's self-quarantine by obtaining their real-time } \\
\text { locations from their mobile phones (e.g., signal) }\end{array}$ & $x$ & $x$ & $\mathrm{O}$ \\
\hline $\mathrm{M} 7$ * & & $\begin{array}{l}\text { Monitoring people's self-quarantine by requiring them to wear } \\
\text { an e-wristband that reported their real-time locations to public } \\
\text { health officers }\end{array}$ & $\mathrm{O}$ & $x$ & $\square$ \\
\hline M8 & & $\begin{array}{l}\text { People were required to carry a valid travel certificate (i.e., not in } \\
\text { self-quarantine) when using public places }\end{array}$ & $\diamond$ & $x$ & $x$ \\
\hline M9 & \multirow{2}{*}{$\begin{array}{l}\text { Location } \\
\text { Disclosure }\end{array}$} & $\begin{array}{l}\text { Publicly disclosing the locations of major activities of COVID-19 } \\
\text { patients with their ages and genders }\end{array}$ & $\mathrm{O}$ & $x$ & $\mathrm{O}$ \\
\hline M10 & & $\begin{array}{c}\text { Publicly disclosing the locations of major activities of COVID-19 } \\
\text { patients (not disclosing ages and genders) }\end{array}$ & $\mathrm{O}$ & $x$ & $\mathrm{O}$ \\
\hline
\end{tabular}

Notes: * Measures based on digital technologies. The status of execution of each method is subject to change, as the COVID-19 pandemic situation is rapidly evolving. O: Being used (in most regions). X: Not being used. $\Delta$ : Some regions/institutions are employing this method. $\square$ : Only people who violate the self-quarantine mandate are required to wear an e-wristband. $\diamond:$ Under discussion.

For each of these ten COVID-19 control measures, participants were asked to provide their views concerning the following: (1) the level of privacy concerns he/she may have with the control measure in question; (2) the level of social benefits he/she thinks would be gained by providing the information requested by the measure in question; and (3) the extent to which the measure in question was acceptable to him/her. The views of the participants on each of these three response items for each of the ten COVID-19 control measures were recorded based on a seven-point scale (from 1 to 7). For example, regarding privacy concerns, " 1 " indicates "not concerned at all", and 4 indicates "neutral", while 7 indicates "very concerned". Regarding participants' perceived social benefits of the measures, "1" indicates "not beneficial at all," while " 7 " indicates "very beneficial". Regarding acceptance, "1" indicates "not acceptable at all", while " 7 " indicates "very acceptable" (note that the response items for privacy concerns, perceived social benefits, and acceptance in 
the pooled data have a Cronbach's alpha of $0.908,0.916$, and 0.901 respectively, indicating that they have good internal consistency).

Section 2 of the survey questionnaire collected data about participants' sociodemographic characteristics, individualist-collectivist orientation, and perception of the COVID19 situation in the study areas. The sociodemographic attributes of participants collected include their gender, age, income, race/ethnicity, educational attainment, and employment status. Data on participants' individualist-collectivist orientation were collected using the 16 items from Triandis and Gelfand's [45] study. These include statements like "I'd rather depend on myself than others", "I rely on myself most of the time; I rarely rely on others", "I often do 'my own thing'", and "My personal identity, independent of others, is very important to me". For these 16 statements, participants responded to the question "How much do you agree with the following statements?" Their responses were measured on a seven-point scale (from 1 to 7 ), where " 1 " stands for "strongly disagree", " 4 " stands for "neutral", and "7" stands for "strongly agree". Note that these 16 items from Triandis and Gelfand's [45] are more concerned with a participant's evaluation of his/her individualist and collectivist orientation rather than his/her assessment of the individualist and collectivist orientation of the study area in which he/she lives (thus, unlike House et al. [46]). Among these 16 response items, we selected the responses to three of them to calculate a collectivist orientation score for each participant [33,45]: "If a coworker gets a prize, I would feel proud", "The well-being of my coworkers is important to me", and "I feel good when I cooperate with others". A participant's collectivist orientation score is derived as a factor score based on confirmatory factor analysis (CFA) on the scores for the three selected responses. A higher collectivist orientation score indicates that a participant has a stronger collectivist orientation. Note that the 16 items in the surveys of Triandis and Gelfand [45] cover both individualist and collectivist orientations. We chose only three of the response items to derive the collectivist orientation score because individualism and collectivism captured by the 16 items are mirror concepts, where a higher collectivist orientation score is strongly associated with a lower individualist orientation score.

\section{Results}

3.1. Privacy Concerns, Perceived Social Benefits, and Acceptance of COVID-19 Control Measures in Hong Kong

We begin our analysis by examining Hong Kong people's privacy concerns, perceived social benefits, and acceptances of the ten COVID-19 control measures (i.e., M1-M10). Table 3 shows the results, which are in line with previous findings from the United States and South Korea [33]. First, people have higher privacy concerns for measures that utilize more sensitive and private information. Second, people have lower privacy concerns for measures perceived to have a higher social benefit. Lastly, measures with higher acceptance are those for which people have lower privacy concerns and higher perceived social benefit. Particularly, Hong Kong people have a lower acceptance rate for contact tracing based on digital location surveillance technologies: mobile phones (M2), credit card usage history (M3), and Bluetooth-based proximity tracing (M4) (the acceptance rate is the percentage of participants who chose 5, 6, and 7 for the acceptance response item for each control measure). Regarding self-quarantine monitoring using digital location surveillance technologies (i.e., wearing an e-wristband [M7] and carrying a valid digital travel certificate [M8]), M7 has a higher acceptance rate than M8. Further, participants privacy concerns for location disclosure measures (i.e., M9 and M10) are lower than most of the other measures, while the acceptance rates for these measures are higher than most of the other measures.

The results have several important public health policy implications when implementing location surveillance measures to control COVID-19 in Hong Kong. First, contact tracing that utilizes digital location tracking technologies, such as mobile phones (M2), credit card usage history (M3), and Bluetooth-based proximity tracing (M4), may not be effective in Hong Kong because of their low acceptance rates. For instance, the Hong Kong Government recently provided an optional COVID-19 digital tracking mobile phone app 
called the LeaveHomeSafe app [47] for the public to record their visits to various venues (e.g., restaurants) and usage of taxis. Before entering a public venue, people can record the venue they visit (including the location) and visiting period using the application or handwriting. However, a journalist found that many people in Hong Kong opted to write down the information rather than installing and using the COVID-19 tracking app on their mobile phones [48].

Table 3. Descriptive statistics of privacy concerns, perceived social benefits, and acceptance of different COVID-19 control measures.

\begin{tabular}{|c|c|c|c|c|c|c|}
\hline \multicolumn{7}{|c|}{ Hong Kong } \\
\hline Type & Methods & $\begin{array}{l}\text { Privacy } \\
\text { Concerns }\end{array}$ & $\begin{array}{c}\text { Perceived Social } \\
\text { Benefits }\end{array}$ & Acceptance & $\begin{array}{l}\text { Acceptance } \\
\text { Rate }\end{array}$ & $\begin{array}{c}\text { Disapproval } \\
\text { Rate }\end{array}$ \\
\hline \multirow{4}{*}{ Contact Tracing } & M1 & $3.01(1.88)$ & $5.18(1.72)$ & $4.96(1.81)$ & 0.62 & 0.20 \\
\hline & M2 & $3.95(2.11)$ & $5.01(1.88)$ & $4.21(2.08)$ & 0.39 & 0.36 \\
\hline & M3 & $4.56(2.07)$ & $3.93(2.08)$ & $3.46(2.08)$ & 0.28 & 0.54 \\
\hline & M4 & $4.12(2.14)$ & $4.45(1.97)$ & $3.85(2.04)$ & 0.34 & 0.44 \\
\hline \multirow{4}{*}{$\begin{array}{l}\text { Self-Quarantine } \\
\text { Monitoring }\end{array}$} & M5 & $2.25(1.53)$ & $5.13(1.87)$ & $5.59(1.64)$ & 0.75 & 0.09 \\
\hline & M6 & $3.54(2.14)$ & $4.97(1.86)$ & $4.43(2.09)$ & 0.48 & 0.31 \\
\hline & M7 & $2.95(1.82)$ & $5.19(1.77)$ & $5.11(1.82)$ & 0.64 & 0.19 \\
\hline & M8 & $4.17(2.43)$ & $3.98(2.26)$ & $3.70(2.46)$ & 0.41 & 0.50 \\
\hline Location & M9 & $3.11(1.82)$ & $5.21(1.59)$ & $4.97(1.66)$ & 0.57 & 0.14 \\
\hline Disclosure & M10 & $2.58(1.68)$ & $5.13(1.73)$ & $5.36(1.68)$ & 0.71 & 0.14 \\
\hline
\end{tabular}

Notes: Standard deviations in parentheses.

The results in Table 3 also indicate that the disapproval rates of digital contact tracing measures M3 and M4 and self-quarantine monitoring using digital travel certificates (M8) are high ( $44 \%$ to $54 \%$ ). Note that the disapproval rate of a control measure is the percentage of participants who chose 1,2, and 3 for the acceptance question for each measure. A possible reason for these low acceptance rates and high disapproval rates of these COVID-19 control measures is perhaps people's low trust in the government. Note that the level of trust in the government for a large number of people in Hong Kong has declined drastically as a result of the introduction of the Fugitive Offenders Amendment Bill (or the Extradition Bill) by the Hong Kong Government and the series of demonstrations in response from March 2019 to January 2020 [43,49]. The Hong Kong survey was conducted from 14 August to 14 September 2020 in the heat of the 2019-2020 protests that involved many large-scale violent confrontations between the protestors and riot police. Specifically, public polls found that the percentage of Hong Kong people who said they trust the government fell from around 50\% in January 2019 to around 20\% in June 2020, reflecting a drastic decline in people's trust in the Hong Kong Government as a result of the introduction of the Bill and months of protests [50,51]. A major consequence of such a low trust level is the low acceptance of the COVID-19 control measures implemented by the Hong Kong Government. Concomitantly, people in Hong Kong have become less willing to share their individual-level georeferenced data (IGD) with the government, and thus are less likely to accept the control measures based on digital contact tracing technologies [52-55].

\subsection{Comparing Privacy Concerns, Perceived Social Benefits, and Acceptance of COVID-19 Control Measures between the Three Study Areas}

In this subsection, we use the Mann-Whitney $U$ test to evaluate the differences in privacy concerns, perceived social benefits, and acceptance of different COVID-19 control measures (i.e., M1-M10) between participants in the three study areas (i.e., the United States, South Korea, and Hong Kong). The Mann-Whitney U test is a nonparametric equivalent of the paired-sample $t$-test that does not require the data to follow normal distributions. We used it because the response data do not follow normal distributions, as the Shapiro-Wilk normality test results reveal [56]. Figures 2 and 3 and Tables 4 and 5 show 
the results of this analysis. Note that the acceptance rate is the percentage of participants who chose 5, 6, and 7 for the acceptance response item for each control measure.

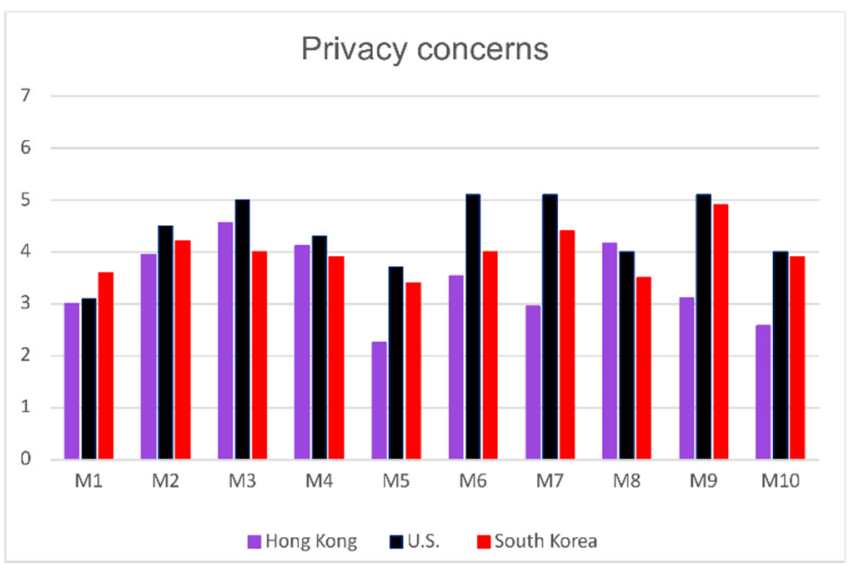

(a)

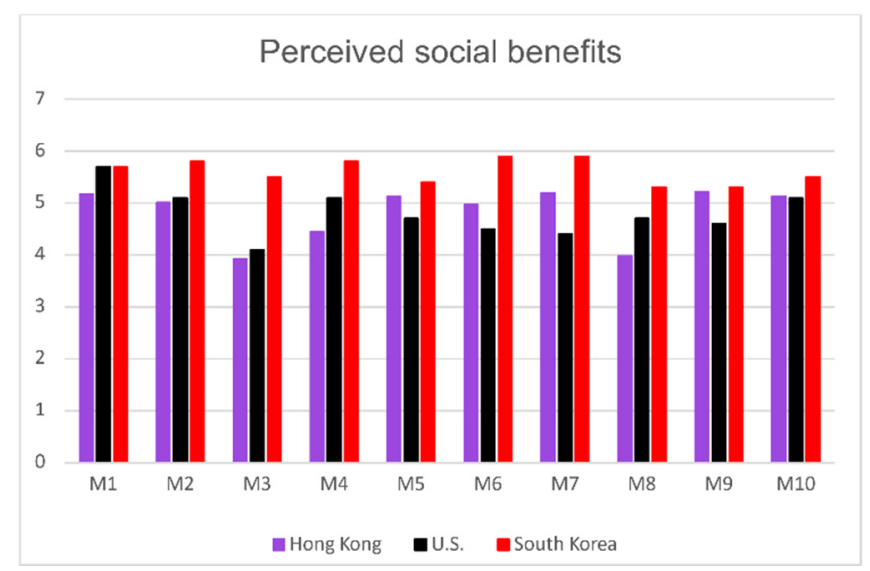

(b)

Figure 2. (a) Average privacy concerns in Hong Kong, the United States, and South Korea; (b) average perceived social benefits in Hong Kong, the United States, and South Korea. Note that M1-M4 are contact tracing measures, M5-M8 are self-quarantine monitoring measures, and M9-M10 are location disclosure measures.

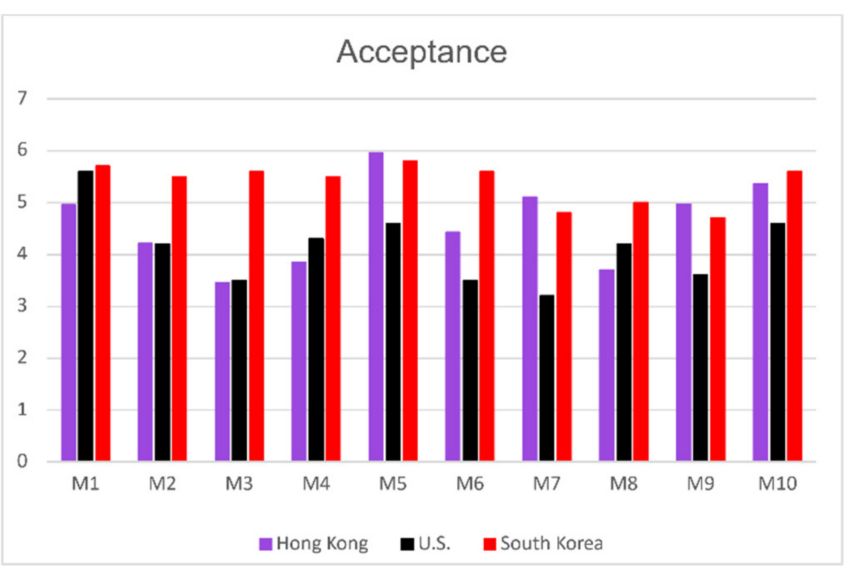

(a)

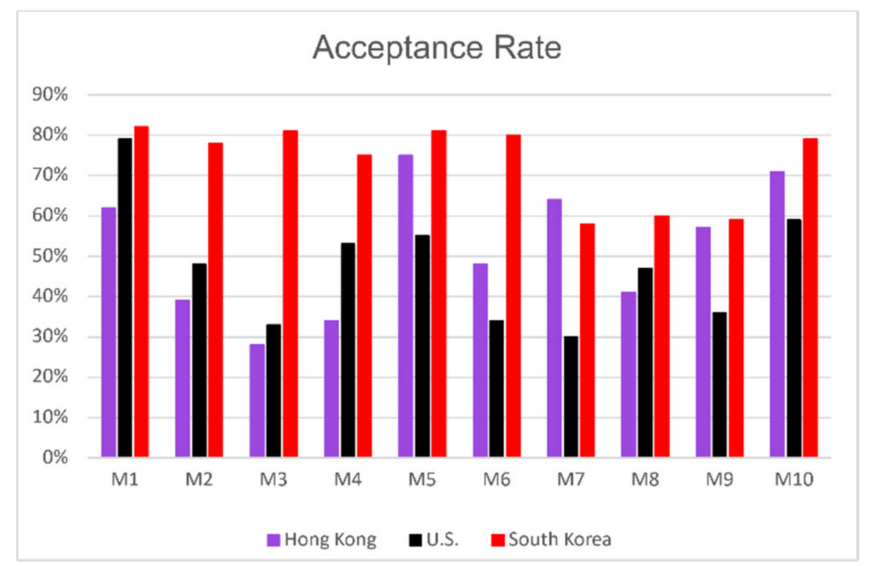

(b)

Figure 3. (a) Average acceptance scores in Hong Kong, the United States, and South Korea; (b) acceptance rates in Hong Kong, the United States, and South Korea. Note that M1-M4 are contact tracing measures, M5-M8 are self-quarantine monitoring measures, and M9-M10 are location disclosure measures.

First, privacy concerns for the contact tracing measures (M1-M4) in the United States, South Korea, and Hong Kong tend to have similar patterns (Figure 2a and Tables 4 and 5). There is a trade-off relationship among privacy concerns, perceived social benefits, and acceptance in the United States and Hong Kong, but not in South Korea (Figure 2a,b and Figure 3a,b); control measures with higher perceived social benefits tend to have lower privacy concerns and higher acceptance, and vice versa. In South Korea, perceived social benefits and acceptance rates are consistently high for all the contact tracing measures. Table 4 shows that the acceptance of contact tracing measures in South Korea and Hong Kong is significantly different (i.e., $p$-value $<0.001$ ), despite similar privacy concern patterns. Table 5 indicates that the privacy concerns for and acceptance of contact tracing measures (except M1) in the United States and Hong Kong are similar (i.e., high privacy concerns and low acceptance). 
Table 4. Mann-Whitney U test results for different COVID-19 control measures (comparison between Hong Kong and South Korea).

\begin{tabular}{|c|c|c|c|c|c|c|c|}
\hline \multicolumn{8}{|c|}{ Hong Kong-South Korea } \\
\hline \multirow[t]{2}{*}{ Type } & \multirow[t]{2}{*}{ Methods } & \multicolumn{2}{|c|}{ Privacy Concerns } & \multicolumn{2}{|c|}{ Perceived Social Benefits } & \multicolumn{2}{|c|}{ Acceptance } \\
\hline & & $p$-value & $|r|$ & $p$-value & $|\mathrm{r}|$ & $p$-value & $|\mathrm{r}|$ \\
\hline \multirow{4}{*}{ Contact Tracing } & M1 & $0.005^{* *}$ & 0.17 & 0.023 & 0.14 & $0.002 * *$ & 0.19 \\
\hline & M2 & 0.438 & 0.05 & $0.001^{* *}$ & 0.20 & $0.000^{* * *}$ & 0.31 \\
\hline & M3 & 0.022 & 0.14 & $0.000^{* * *}$ & 0.38 & $0.000^{* * *}$ & 0.49 \\
\hline & M4 & 0.470 & 0.04 & $0.000^{* * *}$ & 0.33 & $0.000^{* * *}$ & 0.39 \\
\hline \multirow{4}{*}{$\begin{array}{l}\text { Self-Quarantine } \\
\text { Monitoring }\end{array}$} & M5 & $0.000^{* * *}$ & 0.30 & 0.472 & 0.04 & 0.557 & 0.04 \\
\hline & M6 & 0.067 & 0.11 & $0.000 * * *$ & 0.22 & $0.000^{* * *}$ & 0.28 \\
\hline & M7 & $0.000 * * *$ & 0.33 & $0.009^{* *}$ & 0.16 & 0.167 & 0.08 \\
\hline & M8 & 0.024 & 0.14 & $0.000 * * *$ & 0.28 & $0.000^{* * *}$ & 0.26 \\
\hline Location & M9 & $0.000^{* * *}$ & 0.43 & 0.725 & 0.02 & 0.369 & 0.05 \\
\hline Disclosure & M10 & $0.000^{* * *}$ & 0.34 & 0.130 & 0.09 & 0.530 & 0.04 \\
\hline
\end{tabular}

Notes: $\mathrm{r}$ denotes effect size. ${ }^{* * *}$ denotes $p<0.001 .{ }^{* *}$ denotes $p<0.01$.

Table 5. Mann-Whitney U test results for different COVID-19 control methods (comparison between Hong Kong and the United States).

\begin{tabular}{|c|c|c|c|c|c|c|c|}
\hline \multicolumn{8}{|c|}{ Hong Kong-U.S. } \\
\hline \multirow[t]{2}{*}{ Types } & \multirow[t]{2}{*}{ Methods } & \multicolumn{2}{|c|}{ Privacy Concerns } & \multicolumn{2}{|c|}{ Perceived Social Benefits } & \multicolumn{2}{|c|}{ Acceptance } \\
\hline & & $p$-value & $|r|$ & $p$-value & $|r|$ & $p$-value & $|r|$ \\
\hline \multirow{4}{*}{ Contact Tracing } & M1 & 0.826 & 0.01 & $0.000 * * *$ & 0.19 & $0.001 * *$ & 0.17 \\
\hline & M2 & 0.035 & 0.11 & 0.506 & 0.04 & 0.936 & 0.00 \\
\hline & M3 & 0.065 & 0.10 & 0.327 & 0.05 & 0.831 & 0.01 \\
\hline & M4 & 0.388 & 0.05 & $0.000^{* * *}$ & 0.19 & 0.048 & 0.11 \\
\hline \multirow{4}{*}{$\begin{array}{l}\text { Self-Quarantine } \\
\text { Monitoring }\end{array}$} & M5 & $0.000 * * *$ & 0.33 & 0.058 & 0.10 & $0.000^{* * *}$ & 0.24 \\
\hline & M6 & $0.000 * * *$ & 0.35 & 0.045 & 0.11 & $0.000^{* * *}$ & 0.21 \\
\hline & M7 & $0.000 * * *$ & 0.47 & $0.001 * *$ & 0.18 & $0.000^{* * *}$ & 0.45 \\
\hline & M8 & 0.560 & 0.03 & $0.005^{* *}$ & 0.15 & 0.110 & 0.09 \\
\hline Location & M9 & $0.000 * * *$ & 0.48 & $0.009 * *$ & 0.14 & $0.000^{* * *}$ & 0.32 \\
\hline Disclosure & M10 & $0.000^{* * *}$ & 0.35 & 0.919 & 0.01 & $0.000 * * *$ & 0.19 \\
\hline
\end{tabular}

Notes: $\mathrm{r}$ denotes effect size. ${ }^{* * *}$ denotes $p<0.001 .{ }^{* *}$ denotes $p<0.01$.

Second, focusing on the self-quarantine monitoring measures (M5-M8), privacy concerns in Hong Kong are lower than those in the United States and South Korea for M5-M7, but not M8 (Figure 2a). Specifically, people in Hong Kong have the lowest privacy concerns for M5 and the highest privacy concerns for M8, which is in contrast with the United States and South Korea (where privacy concerns for M8 are relatively low). Thus, people in Hong Kong have the highest acceptance for M5 and the lowest acceptance for M8 (Figure 3a,b). Further, as shown in Table 4, privacy concerns for M5 and M7 and acceptance of M8 are significantly different (i.e., $p$-value $<0.001$ ) between Hong Kong and South Korea. Table 5 shows that the privacy concerns for and acceptance of M5-M7 are significantly different (i.e., $p$-value $<0.001$ ) between Hong Kong and the United States.

Third, Figure 2a indicates that people in Hong Kong have distinctively lower privacy concerns than people in the United States and South Korea for the two location disclosure measures (M9 and M10). Further, as shown in Tables 4 and 5, privacy concerns for M9 and M10 are significantly different $(p$-value $<0.001)$ between Hong Kong and the United States as well as between Hong Kong and South Korea. Meanwhile, acceptance for M9 and M10 is significantly different between Hong Kong and the United States. These results suggest that, although people in South Korea and Hong Kong have higher acceptance for M9 and M10 than the United States, it may be because of different considerations. For instance, 
people in Hong Kong have higher acceptance of M9 and M10 as they have lower privacy concerns for those measures. However, people in South Korea have higher acceptance for the M9 and M10 despite that their privacy concerns for these two measures are similar to that in the United States. In other words, people in South Korea worry about their privacy, but are more willing to accept these two control measures (M9 and M10), perhaps because of the high perceived social benefits of these measures, which may in turn be because of the stronger collectivist orientation of the people in South Korea.

These results have several important implications for public health policies that seek to control the COVID-19 pandemic. First, people's acceptance of COVID-19 control measures can be significantly improved by increasing people's perceived social benefits based on their experiences of past epidemics, even when they have high privacy concerns for those measures. For instance, people in South Korea believe that digital contact tracing measures (e.g., M2, M3, and M4) and the disclosure of personal locations are vital to flatten the curve of confirmed cases and reduce deaths [57,58]. Their collective experience of the Middle East Respiratory Syndrome (MERS) outbreak in 2015 triggered the amendment of the Contagious Disease Prevention and Control Act (CDPCA), which allows public health authorities to collect, use, and disclose personal locations, despite that doing so may violate personal privacy $[57,58]$. On the other hand, people in Hong Kong also have collectively experienced the Severe Acute Respiratory Syndrome (SARS) in 2003, which has underlined the need for better and early identification and isolation of imported cases to contain the pandemic [42]. Hence, people in Hong Kong share a consensus on the importance of implementing self-quarantine for inbound travelers through e-wristband (e.g., M7) and location disclosure of infected persons (e.g., M9 and M10) for controlling the COVID-19 pandemic.

Second, our results indicate that people in Hong Kong and the United States have low acceptance of self-quarantine monitoring that uses digital travel certificates (M8) as their privacy concerns for digital travel certificates are high. However, the results do not necessarily imply that Hong Kong and the U.S. cannot implement self-quarantine via travel certificates. Specifically, with COVID-19 vaccination accelerating, the Hong Kong and the U.S. Governments could issue travel or vaccination certificates for people to indicate their vaccination status and to allow them to enter certain public venues (e.g., museums, libraries, universities, bars, and restaurants). Considering the privacy concerns of people in Hong Kong and the United States for the digital travel certificates, travel certificates can be implemented in either paper or digital form. For instance, a physical "yellow card" created by the World Health Organization (WHO) has been used in Hong Kong [59] for decades by international travelers to show their inoculation against yellow fever. Further, the New York State Government has become the first state in the United States to implement a "Vaccine Passport" system [60], which some venues have used to identify people's vaccination status.

\subsection{Associations Between Sociodemographic Characteristics and Privacy Concerns, Perceived Social Benefits, and Acceptance of COVID-19 Control Measures}

In this subsection, we further explore the associations between people's privacy concerns, perceived social benefits, and acceptance of the ten COVID-19 control measures and their sociodemographic characteristics using linear regression. The dependent variables of the regression models are the aggregated acceptance score (Model 1), aggregated privacy concern score (Model 2), and aggregated perceived social benefit score (Model 3), which were obtained by adding each score of the acceptance, privacy concern, and perceived social benefit response items for the ten control measures (M1-M10). A higher score indicates that a person has higher privacy concerns, perceived social benefits, and acceptance for the ten COVID-19 control measures. As each response item in the surveys was measured on a scale of 1 to 7 , the minimum value of these three dependent variables is 10 (10 items $\left.{ }^{*} 1\right)$, while the maximum value is 70 (10 items* 7$)$. For the aggregated acceptance score, the average score is 46.3 , the median score is 46 , and the standard deviation is 14.4. For the aggregated privacy concern score, the average score is 38.9 , the median score is 41 , and the standard 
deviation is 13.7. For the aggregated perceived social benefit score, the average score is 50.6, the median score is 52 and the standard deviation is 13.7. The independent variables of the three regression models include gender (female: 1; male: 0 ), age group 1 (18-24 years old: $1 ; 35-44$ years old: 0$)$, age group 2 (45+ years old: $1 ; 35-44$ years old: 0$)$, student (yes: 1 ; no: 0), employed (yes: 1; no: 0), higher education (yes: 1, no: 0 ), the collectivist orientation score, and the residing countries/regions: South Korea (South Korea: 1; the United States and Hong Kong: 0) and the United States (the United States: 1; Hong Kong and South Korea: 0).

Table 6 shows the regression results for Models 1-3. The results of Model 1 show that people in South Korea (compared to those in Hong Kong and the U.S.) and people with higher collectivist orientation scores tend to have higher acceptance scores $(p<0.001)$. The results of Model 2 indicate that people having higher collectivist orientation scores are significantly associated with lower privacy concerns. When compared with males, female respondents have higher privacy concerns $(p<0.01)$. Further, people in the United States (compared with those in Hong Kong and South Korea) are significantly associated with higher privacy concern $(p<0.01)$, while people in Hong Kong (compared with those in the United States and South Korea) are significantly associated with lower privacy concern $(p<0.01)$. The results of Model 3 suggest that people with higher collectivist orientation scores and higher education degrees are significantly associated with higher perceived social benefits $(p<0.001$ and $p<0.05)$. Meanwhile, people in Hong Kong and the United States (compared with those in South Korea) are significantly associated with lower perceived social benefits $(p<0.01)$.

Table 6. Results of the linear regression models for individuals' acceptance (Model 1), privacy concerns (Model 2), and perceived social benefits (Model 3) in the United States, South Korea, and Hong Kong $(n=433)$.

\begin{tabular}{|c|c|c|c|c|}
\hline \multicolumn{2}{|c|}{ Variables } & $\begin{array}{c}\text { Model 1 } \\
\text { (Acceptance) }\end{array}$ & $\begin{array}{c}\text { Model } 2 \\
\text { (Privacy Concerns) }\end{array}$ & $\begin{array}{c}\text { Model } 3 \\
\text { (Perceived Social Benefits) }\end{array}$ \\
\hline \multicolumn{2}{|c|}{ Female } & $-0.084(0.095)$ & $0.263(0.098)^{* *}$ & $-0.161(0.099)$ \\
\hline Age & $\begin{array}{l}\text { Age } 1(18-24) \\
\text { Age } 2(45+)\end{array}$ & $\begin{array}{l}-0.153(0.119) \\
0.083(0.123)\end{array}$ & $\begin{array}{c}0.133(0.123) \\
-0.080(0.128)\end{array}$ & $\begin{array}{c}-0.067(0.123) \\
0.037(0.128)\end{array}$ \\
\hline Employment Status & $\begin{array}{c}\text { Student } \\
\text { Employed }\end{array}$ & $\begin{array}{l}0.107(0.125) \\
0.077(0.105)\end{array}$ & $\begin{array}{l}-0.117(0.130) \\
-0.009(0.109)\end{array}$ & $\begin{array}{l}0.031(0130) \\
0.094(0.109)\end{array}$ \\
\hline \multicolumn{2}{|c|}{ Higher education } & $0.035(0.177)$ & $-0.006(0.122)$ & $0.281(0.122) *$ \\
\hline $\begin{array}{l}\text { Country/ } \\
\text { Region }{ }^{1}\end{array}$ & $\begin{array}{c}\text { USA } \\
\text { South Korea }\end{array}$ & $\begin{array}{l}-0.430(0.108)^{* * *} \\
0.586(0.120)^{* * *}\end{array}$ & $\begin{array}{l}0.724(0.125) * * * \\
0.398(0.124)\end{array}$ & $\begin{array}{l}-0.109(0.112)^{* * * *} \\
0.573(0.124)^{* * *}\end{array}$ \\
\hline \multicolumn{2}{|c|}{ Collectivist orientation score } & $0.223(0.048)^{* * *}$ & $-0.186(0.049)^{* * *}$ & $0.180(0.049)^{* * *}$ \\
\hline \multicolumn{2}{|c|}{ Intercept } & $-0.002(0.176)$ & $-0.530(0.182)^{* *}$ & $-0.278(0.182)$ \\
\hline \multicolumn{2}{|c|}{$\mathrm{R}^{2}$} & $\begin{array}{l}0.175 \\
0.157\end{array}$ & $\begin{array}{l}0.120 \\
0.101\end{array}$ & $\begin{array}{l}0.118 \\
0.099\end{array}$ \\
\hline
\end{tabular}

Notes: Standard errors in parentheses; ${ }^{* * *}$ denotes $p<0.001 ;{ }^{* *}$ denotes $p<0.01 ;{ }^{*}$ denotes $p<0.05$. ${ }^{1}$ Reference: Hong Kong.

Further, to better understand the role of age and gender in people's privacy concerns, perceived social benefits, and acceptance of the COVID-19 control measures, we investigated the age and gender difference in the aggregated privacy concern scores, the aggregated perceived social benefits scores, and the aggregated acceptance scores among the three study areas (Figures 4 and 5). Figure 4 shows that age differences in the aggregated privacy concern scores, the aggregated perceived social benefits scores, and the aggregated acceptance scores are larger in Hong Kong than in the United States and South Korea. Meanwhile, young people (i.e., age < 24) in Hong Kong and South Korea have higher privacy concerns for and lower acceptance of the COVID-19 control measures than people who are older than 24, which is contrary to the United States. Further, the results also suggest that young people in Hong Kong, South Korea, and the United States have lower perceived social benefits for the COVID-19 control measures than people who are older than 24. 


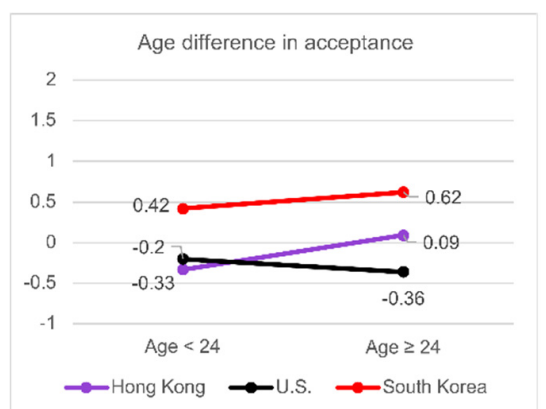

(a)

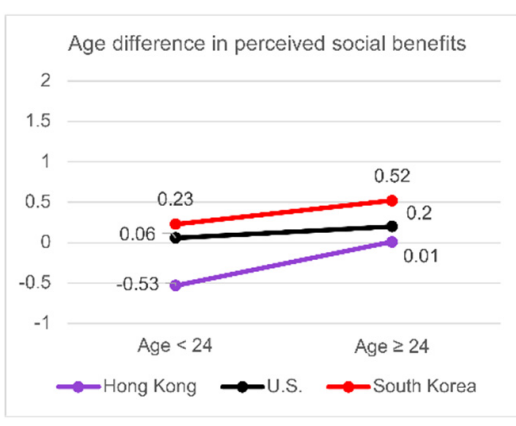

(b)

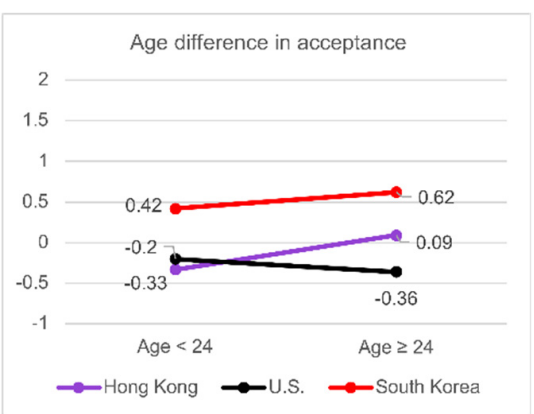

(c)

Figure 4. Age differences in people's privacy concerns, perceived social benefits, and acceptance for the COVID-19 control measures in Hong Kong, the United States, and South Korea: (a) average aggregated privacy concern scores (standardized); (b) average aggregated perceived social benefits scores (standardized); (c) average aggregated acceptance scores (standardized).

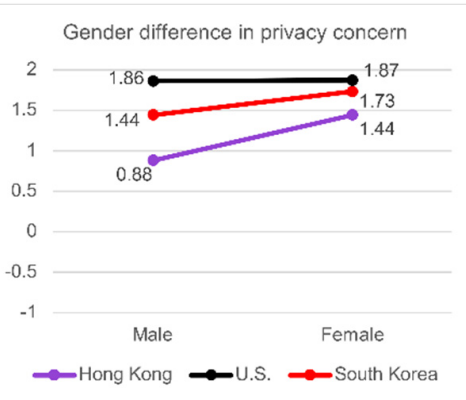

(a)

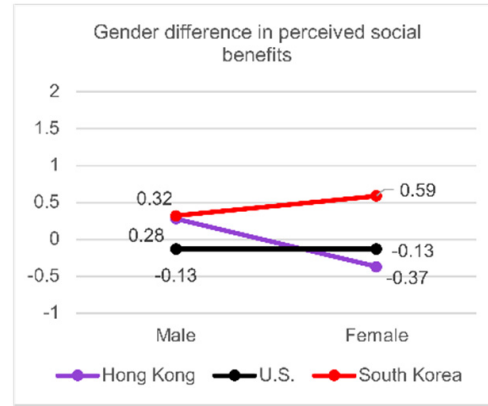

(b)

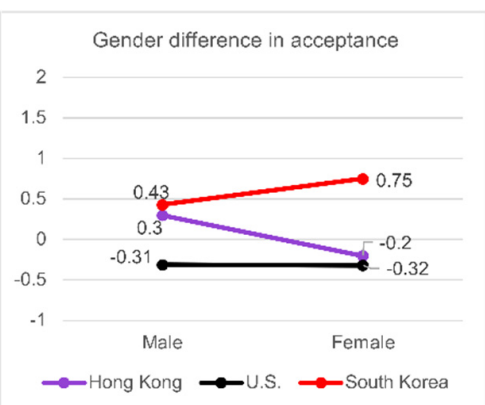

(c)

Figure 5. Gender differences in people's privacy concerns, perceived social benefits, and acceptance for the COVID-19 control measures in Hong Kong, the United States, and South Korea: (a) average aggregated privacy concern scores (standardized); (b) average aggregated perceived social benefits scores (standardized); (c) average aggregated acceptance scores (standardized).

Figure 5 shows that gender differences in the aggregated privacy concern scores, the aggregated perceived social benefits scores, and the aggregated acceptance scores are larger in Hong Kong than in the United States and South Korea. First, in the United States, men and women have the same aggregated privacy concern scores, aggregated perceived social benefits scores, and aggregated acceptance scores. Second, gender differences in the aggregated privacy concern scores, aggregated perceived social benefits scores, and aggregated acceptance scores are larger in Hong Kong than in the United States and South Korea. Further, in Hong Kong and South Korea, women have higher privacy concerns than men. Meanwhile, women in Hong Kong have lower perceived social benefits and acceptance for the COVID-19 control measures than men, which is contrary to South Korea.

These results indicate that age and gender may be important factors in some areas (e.g., Hong Kong and South Korea) in influencing people's privacy concerns, perceived social benefits, and acceptance of the COVID-19 control measures. In other words, there is geographic heterogeneity in the age and gender differences in people's privacy concerns, perceived social benefits and acceptance for the COVID-19 control measures: different countries or regions may have different patterns of age and gender differences in people's privacy concerns, perceived social benefits, and acceptance for the COVID-19 control measures.

These results lead to several important observations. First, individuals with a stronger collectivist orientation tend to have higher perceived social benefits for COVID-19 control measures, which is consistent with previous findings $[33,61]$. Further, a person's collectivist orientation strengthens the positive relationship between his/her perceived social benefits and acceptance of the control measures $[62,63]$. Thus, individuals with a stronger collec- 
tivist orientation tend to be more willing to accept COVID-19 control measures that are perceived to provide significant social benefits despite the privacy threat. These results are consistent with findings from other studies that have investigated the relationships among people's collectivist orientation, perceived social benefits, and acceptances of different things (e.g., people's opinions on green purchase behavior, bicycle-sharing, and influenza vaccination) [64-66].

Second, we found that women in Hong Kong and South Korea have higher privacy concerns for the COVID-19 control measures than men. Further, the differences in the aggregated privacy concern scores, aggregated perceived social benefits scores, and aggregated acceptance scores between men and women are larger in Hong Kong than in South Korea. One explanation is that female COVID-19 patients may suffer a higher level of threat such as cyberbullying or cyber violence (e.g., lifestyle shaming) than male COVID-19 patients after their personal information and visited locations are disclosed to the public. For instance, women's lifestyles were often criticized in South Korea and China on social media platforms (e.g., Weibo and Twitter) after the venues or locations they visited and their activities were published or after their personal data were leaked $[67,68]$.

Third, our results suggest that people's residing areas play an important role in their privacy concerns, perceived social benefits, and acceptance of COVID-19 control measures. For instance, people in Hong Kong have similar patterns of privacy concerns for and acceptances of digital contact tracing measures (i.e., M2, M3, and M4) to people in the United States. Meanwhile, people in Hong Kong also have similar patterns of acceptance for the two location disclosure measures (M9 and M10) to people in South Korea, despite the patterns of privacy concerns for these two measures being different between these two study areas. The explanations for these results include the following: (1) The low level of trust of Hong Kong people in their government (as mentioned in Section 3.1) would lead to higher privacy concerns, which would then make them less likely to accept the control measures based on digital contact tracing. (2) Hong Kong people's (especially the younger generation) individualism has increased (i.e., collectivism has decreased) over the past several decades related to the globalization of individualist thinking (Figure 6) $[69,70]$. Increasing individualism among the people in Hong Kong would thus heighten their privacy concerns and reduce their acceptance of the COVID-19 control measures in Hong Kong. (3) People in Hong Kong and South Korea have similar collective memories for the SARS in 2003 and the MERS and 2015. Their epidemic experiences led them to think that timely disclosure of the personal location information of infected persons to the public would yield important social benefits during a pandemic. Further, it should be noted that people in the United States did not have any recent collective experiences of serious epidemics like the SARS or the MERS. Thus, people in Hong Kong and South Korea have similar patterns of acceptance for the two location disclosure methods (M9 and M10).

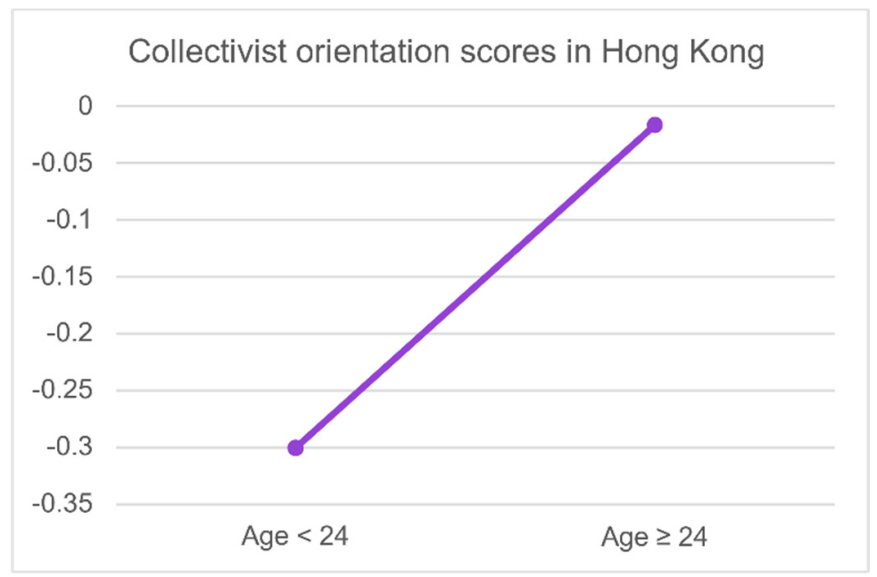

Figure 6. The average collectivist orientation scores (standardized) in Hong Kong. 


\section{Conclusions and Discussion}

This study first analyzed data collected from 149 respondents from Hong Kong to understand people's privacy concerns, perceived social benefits, and acceptance of ten COVID-19 control measures that use individual-level georeferenced data (IGD). Then, we compared the views of the Hong Kong participants with those of a U.S. sample (188 respondents) and a South Korean sample (118 respondents) obtained in an earlier study by Kim and Kwan [33]. Lastly, we investigated the associations between people's views on COVID-19 control measures and their sociodemographic characteristics using linear regression. The results indicate that people's views on COVID-19 control measures are different across Hong Kong, the United States, and South Korea. These results provide important insights for policymakers in developing control measures for COVID-19 and future epidemics.

First, the results revealed that people in Hong Kong have a lower acceptance rate for contact tracing measures that use digital location surveillance technologies (M2, M3, and M4), which may be because of their low trust in the government. The low public trust in the government would make people less willing to share their individual-level georeferenced data (IGD), and thus less likely to accept the COVID-19 control measures based on digital contact tracing technologies. In other words, promoting public trust between people and the government might be critical when trying to control the spread of a highly contagious infectious disease like COVID-19. Hence, policymakers should seek to address people's low trust in government when developing intervention measures.

Second, our results suggest that people's collective experience of previous epidemics may make them more willing to accept certain control measures despite those measures being perceived to pose a considerable privacy threat. For instance, both Hong Kong and South Korea have a recent collective experience of an epidemic (the SARS in 2003 for the former and the MERS in 2015 for the latter), which has highlighted the need for the early identification and isolation of imported cases, contact tracing, and public disclosure of the locations visited by infected persons for controlling the spread of the COVID-19 pandemic. Thus, people in Hong Kong can accept self-quarantine monitoring that uses an e-wristband for inbound travelers and the timely disclosure of the locations visited by COVID-19 patients to the public. Further, people in South Korea can accept control measures that use digital location contact tracing.

Lastly, our results indicate that there is geographic heterogeneity in the age and gender differences in people's privacy concerns, perceived social benefits, and acceptance for the COVID-19 control measures; that is, different countries or regions may have different patterns of age and gender differences in people's privacy concerns, perceived social benefits, and acceptance for the COVID-19 control measures. Specifically, age and gender differences in privacy concerns, perceived social benefits, and acceptance for COVID-19 control measures are larger in Hong Kong and South Korea than in the United States. Moreover, people in Hong Kong have the largest age and gender differences in privacy concerns, perceived social benefits, and acceptance for the COVID-19 measures among the three study areas. Thus, policymakers should pay more attention to specific social groups as they may have greater privacy concerns. For example, women in Hong Kong and South Korea have greater privacy concerns than men, which may be because women tend to suffer a higher level of cyberbullying or cyber violence (e.g., lifestyle shaming) than men after their personal information or historical locations are disclosed to the public. In this light, these specific groups call for particular attention in the design of COVID-19 control measures.

However, our research has several limitations that future studies should address. First, the samples of the study obtained via online surveys are biased toward younger and highly educated people for the three study areas. Further, the United States and Hong Kong samples have a higher percentage of women than men, while the South Korean sample has a higher percentage of men than women. It is thus important to bear in mind such a sampling bias in our datasets and be cautious when interpreting the results, especially 
not to speak about the experiences of the participants as if they are representative of the "people" of a study area. Further, the gender categories used in our surveys are limited to only male and female, which ignore non-binary gender categories (i.e., LGBTQ+) and the experiences of people in these categories. Meanwhile, the error margins of the three samples are 8\%, 7\%, and 9\% (Hong Kong, the United States, and South Korea) at the $95 \%$ confidence level. Future studies would benefit from using or including other survey methods (e.g., telephone surveys) to obtain more representative samples. Second, future studies should include other potentially important factors such as trust in government and the use of incentives to increase people's acceptance of various control measures. For instance, Guillon and Kergall [71] found that trust in government is highly positively associated with the acceptance of digital contact tracing apps in France. Munzert et al. [72] observed that even small monetary incentives could strongly increase the acceptance of digital contact tracing apps in Germany. Lastly, it should be noted that people's views on COVID-19 control measures are dynamic and can change over time, particularly during the pandemic. At the time of writing (i.e., June 2021), the United States, South Korea, Hong Kong are at different stages of vaccination deployment, and their residents hold widely varying attitudes toward vaccination. Such differences may be related to and influence people's views on COVID-19 control measures throughout the pandemic, which future studies need to continually monitor.

Author Contributions: Jianwei Huang, Mei-Po Kwan, and Junghwan Kim conceived the idea; Junghwan Kim and Mei-Po Kwan designed the online surveys; Jianwei Huang, Junghwan Kim, and Mei-Po Kwan conducted the online surveys; Jianwei Huang analyzed the data and wrote and revised the paper; Mei-Po Kwan contributed to writing and revising the paper; Junghwan Kim contributed to revising the paper. All authors have read and agreed to the published version of the manuscript.

Funding: Part of this research was supported by grants from the Hong Kong Research Grants Council (General Research Fund Grant no. 14605920, 14611621; Collaborative Research Fund Grant no. C4023-20GF) and a grant from the Research Committee on Research Sustainability of Major Research Grants Council Funding Schemes of the Chinese University of Hong Kong.

Institutional Review Board Statement: The study was conducted according to the guidelines of the Declaration of Helsinki. The U.S. and South Korean surveys were approved by the Institutional Review Board of the University of Illinois at Urbana-Champaign (IRB Approval No. 20974; 25 June 2020). The Hong Kong survey was approved by the Survey and Behavioural Research Ethics Committee of the Chinese University of Hong Kong (Approval No. SBRE-19-801; 11 August 2020).

Informed Consent Statement: Informed consent was obtained from all participants involved in the study.

Acknowledgments: The authors would like to thank the participants for their participation in the study and the anonymous reviewers for their helpful comments during the COVID-19 pandemic.

Conflicts of Interest: The authors declare no conflict of interest.

\section{References}

1. Anderson, R.M.; Heesterbeek, H.; Klinkenberg, D.; Hollingsworth, T.D. How will country-based mitigation measures influence the course of the COVID-19 epidemic? Lancet 2020, 395, 931-934. [CrossRef]

2. Davies, N.G.; Kucharski, A.J.; Eggo, R.M.; Gimma, A.; Edmunds, W.J.; Jombart, T.; O’Reilly, K.; Endo, A.; Hellewell, J.; Nightingale, E.S.; et al. Effects of non-pharmaceutical interventions on COVID-19 cases, deaths, and demand for hospital services in the UK: A modelling study. Lancet Public Health 2020, 5, e375-e385. [CrossRef]

3. Kucharski, A.J.; Klepac, P.; Conlan, A.J.; Kissler, S.M.; Tang, M.L.; Fry, H.; Gog, J.R.; Edmunds, W.J.; Emery, J.C.; Medley, G.; et al. Effectiveness of isolation, testing, contact tracing, and physical distancing on reducing transmission of SARS-CoV-2 in different settings: A mathematical modelling study. Lancet Infect. Dis. 2020, 20, 1151-1160. [CrossRef]

4. Bradshaw, W.J.; Alley, E.C.; Huggins, J.H.; Lloyd, A.L.; Esvelt, K.M. Bidirectional contact tracing could dramatically improve COVID-19 control. Nat. Commun. 2021, 12, 232. [CrossRef]

5. Kan, Z.; Kwan, M.-P.; Wong, M.S.; Huang, J.; Liu, D. Identifying the space-time patterns of COVID-19 risk and their associations with different built environment features in Hong Kong. Sci. Total Environ. 2021, 772, 145379. [CrossRef]

6. Buckee, C.O.; Balsari, S.; Chan, J.; Crosas, M.; Dominici, F.; Gasser, U.; Grad, Y.H.; Grenfell, B.; Halloran, M.E.; Kraemer, M.U.; et al. Aggregated mobility data could help fight COVID-19. Science 2020, 368, 145-146. [CrossRef] 
7. Budd, J.; Miller, B.S.; Manning, E.M.; Lampos, V.; Zhuang, M.; Edelstein, M.; Rees, G.; Emery, V.C.; Stevens, M.M.; Keegan, N.; et al. Digital technologies in the public-health response to COVID-19. Nat. Med. 2020, 26, 1183-1192. [CrossRef] [PubMed]

8. Walrave, M.; Waeterloos, C.; Ponnet, K. Adoption of a contact tracing app for containing COVID-19: A health belief model approach. JMIR Public Health Surveill. 2020, 6, e20572. [CrossRef]

9. Whitelaw, S.; Mamas, M.A.; Topol, E.; Van Spall, H.G. Applications of digital technology in COVID-19 pandemic planning and response. Lancet Digit. Health 2020, 2, e435-e440. [CrossRef]

10. Mbunge, E.; Akinnuwesi, B.; Fashoto, S.G.; Metfula, A.S.; Mashwama, P. A critical review of emerging technologies for tackling COVID-19 pandemic. Hum. Behav. Emerg. Technol. 2021, 3, 25-39. [CrossRef]

11. Ekong, I.; Chukwu, E.; Chukwu, M. COVID-19 mobile positioning data contact tracing and patient privacy regulations: Exploratory search of global response strategies and the use of digital tools in Nigeria. JMIR mHealth uHealth 2020, 8, e19139. [CrossRef]

12. Oliver, N.; Lepri, B.; Sterly, H.; Lambiotte, R.; Deletaille, S.; De Nadai, M.; Letouzé, E.; Salah, A.A.; Benjamins, R.; Cattuto, C.; et al. Mobile phone data for informing public health actions across the COVID-19 pandemic life cycle. Sci. Adv. 2020, 6, eabc0764. [CrossRef]

13. Smith, C.; Mennis, J. Incorporating geographic information science and technology in response to the COVID-19 pandemic. Prev. Chronic Dis. 2020, 17, 200246. [CrossRef]

14. Wu, Z.; McGoogan, J.M. Characteristics of and important lessons from the coronavirus disease 2019 (COVID-19) outbreak in China: Summary of a report of 72,314 cases from the Chinese center for disease control and prevention. JAMA 2020, 323, 1239-1242. [CrossRef]

15. Armstrong, M.P.; Ruggles, J.J. Geographic information technologies and personal privacy. Cartographica 2005, 40, 63-73. [CrossRef]

16. Brownstein, J.S.; Cassa, C.A.; Kohane, I.S.; Mandl, K.D. An unsupervised classification method for inferring original case locations from low-resolution disease maps. Int. J. Health Geogr. 2006, 5, 56. [CrossRef]

17. Brownstein, J.S.; Cassa, C.A.; Mandl, K.D. No place to hide-Reverse identification of patients from published maps. N. Engl. J. Med. 2006, 355, 1741-1742. [CrossRef]

18. Curtis, A.; Mills, J.W.; Agustin, L.; Cockburn, M. Confidentiality risks in fine scale aggregations of health data. Comput. Environ. Urban Syst. 2011, 35, 57-64. [CrossRef]

19. Curtis, A.J.; Mills, J.W.; Leitner, M. Spatial confidentiality and GIS: Re-engineering mortality locations from published maps about hurricane Katrina. Int. J. Health Geogr. 2006, 5, 44. [CrossRef]

20. Capano, G.; Woo, J.J. Designing policy robustness: Outputs and processes. Policy Soc. 2018, 37, 422-444. [CrossRef]

21. Bavel, J.J.V.; Baicker, K.; Boggio, P.S.; Willer, R. Using social and behavioural science to support COVID-19 pandemic response. Nat. Hum. Behav. 2020, 4, 460-471. [CrossRef]

22. Habersaat, K.B.; Betsch, C.; Danchin, M. Ten considerations for effectively managing the COVID-19 transition. Nat. Hum. Behav. 2020, 4, 677-687. [CrossRef]

23. Ding, Y.; Du, X.; Li, Q.; Zhang, M.; Zhang, Q.; Tan, X.; Liu, Q. Risk perception of coronavirus disease 2019 (COVID-19) and its related factors among college students in China during quarantine. PLoS ONE 2020, 15, e0237626. [CrossRef]

24. de Bruin, W.B.; Bennett, D. Relationships between initial COVID-19 risk perceptions and protective health behaviors: A national survey. Am. J. Prev. Med. 2020, 59, 157-167. [CrossRef]

25. Dryhurst, S.; Schneider, C.R.; Kerr, J.; Freeman, A.L.J.; Recchia, G.; van der Bles, A.M.; Spiegelhalter, D.; van der Linden, S. Risk perceptions of COVID-19 around the world. J. Risk Res. 2020, 23, 994-1006. [CrossRef]

26. Gelfand, M.J.; Jackson, J.C.; Pan, X.; Nau, D.; Pieper, D.; Denison, E.; Dagher, M.; Van Lange, P.A.; Chiu, C.Y.; Wang, M. The relationship between cultural tightness-looseness and COVID-19 cases and deaths: A global analysis. Lancet Planet. Health 2021, 5, e135-e144. [CrossRef]

27. Lu, N.; Cheng, K.W.; Qamar, N.; Huang, K.C.; Johnson, J.A. Weathering COVID-19 storm: Successful control measures of five Asian countries. Am. J. Infect. Control. 2020, 48, 851-852. [CrossRef] [PubMed]

28. Yu, X.; Wong, M.S.; Kwan, M.P.; Nichol, J.E.; Zhu, R.; Heo, J.; Chan, P.W.; Chin, D.C.W.; Kwok, C.Y.T.; Kan, Z. COVID-19 infection and mortality: Association with PM2.5 concentration and population density-An exploratory study. ISPRS Int. J. Geo-Inf. 2021, 10, 123. [CrossRef]

29. An, B.Y.; Tang, S.Y. Lessons from COVID-19 responses in East Asia: Institutional infrastructure and enduring policy instruments. Am. Rev. Public Adm. 2020, 50, 790-800. [CrossRef]

30. Cha, V. Asia's COVID-19 lessons for the west: Public goods, privacy, and social tagging. Wash. Q. 2020, 43, 1-18. [CrossRef]

31. France Weighs Its Love of Liberty in Fight Against Coronavirus. Available online: https://www.nytimes.com/2020/04/17 / world/europe/coronavirus-france-digital-tracking.html (accessed on 22 February 2021).

32. Yan, B.; Zhang, X.; Wu, L.; Zhu, H.; Chen, B. Why do countries respond differently to COVID-19? A comparative study of Sweden, China, France, and Japan. Am. Rev. Public Adm. 2020, 50, 762-769. [CrossRef]

33. Kim, J.; Kwan, M.-P. An examination of people's privacy concerns, perceptions of social benefits, and acceptance of COVID-19 mitigation measures that harness location information: A comparative study of the USA and South Korea. ISPRS Int. J. Geo Inf. 2021, 10, 25. [CrossRef]

34. Kim, J.; Kwan, M.-P.; Levenstein, M.C.; Richardson, D.B. How do people perceive the disclosure risk of maps? Examining the perceived disclosure risk of maps and its implications for geoprivacy protection. Cartogr. Geogr. Inf. Sci. 2021, 48, 2-20. [CrossRef] 
35. Lewis, D. Why many countries failed at COVID contact-tracing-But some got it right. Nature 2020, 588, 384-387. [CrossRef]

36. Shaw, R.; Kim, Y.K.; Hua, J. Governance, technology and citizen behavior in pandemic: Lessons from COVID-19 in East Asia. Prog. Disaster Sci. 2020, 6, 100090. [CrossRef]

37. Hofstede Insights. Available online: https://www.hofstede-insights.com/country-comparison/hong-kong, south-korea, the-usa/ (accessed on 12 July 2021).

38. Huff, L.; Kelley, L. Levels of organizational trust in individualist versus collectivist societies: A seven-nation study. Organ. Sci. 2003, 14, 81-90. [CrossRef]

39. Huang, J.; Kwan, M.-P.; Kan, Z.; Wong, M.S.; Kwok, C.Y.T.; Yu, X. Investigating the relationship between the built environment and relative risk of COVID-19 in Hong Kong. ISPRS Int. J. Geo-Inf. 2020, 9, 624. [CrossRef]

40. The Threat That COVID-19 Poses Now. Available online: https://www.theatlantic.com/health/archive/2021/04/fourth-surgecovid-19-unequal/618493/ (accessed on 12 July 2021).

41. S. Korea's COVID-19 Cases Spike as Delta Variant Spreads. Available online: https://www.reuters.com/world/asia-pacific/ skorea-reports-over-800-covid-19-cases-highest-daily-count-since-jan-7-yonhap-2021-07-01/ (accessed on 12 July 2021 ).

42. Hung, L.S. The SARS epidemic in Hong Kong: What lessons have we learned? J. R. Soc. Med. 2003, 96, 374-378. [CrossRef]

43. Hartley, K.; Jarvis, D.S. Policymaking in a low-trust state: Legitimacy, state capacity, and responses to COVID-19 in Hong Kong. Policy Soc. 2020, 39, 403-423. [CrossRef]

44. Hibberts, M.; Johnson, R.B.; Hudson, K. Common survey sampling techniques. In Handbook of Survey Methodology for the Social Sciences; Gideon, L., Ed.; Springer: New York, NY, USA, 2012; pp. 53-74.

45. Triandis, H.C.; Gelfand, M.J. Converging measurement of horizontal and vertical individualism and collectivism. J. Personal. Soc. Psychol. 1998, 74, 118-128. [CrossRef]

46. House, R.J.; Hanges, P.J.; Javidan, M.; Dorfman, P.W.; Gupta, V. Culture, Leadership, and Organizations: The GLOBE Study of 62 Societies; Sage: London, UK, 2004.

47. LeaveHomeSafe. Available online: https://www.leavehomesafe.gov.hk/en/ (accessed on 17 July 2021).

48. COVID-19: Some Hongkongers Shun Gov't Tracking App Over Privacy Concerns as New Rules Rolled Out at Eateries. Available online: https:/ / hongkongfp.com/2021/02/19/covid-19-some-hongkongers-shun-govt-tracking-app-over-privacy-concernsas-new-rules-rolled-out-at-eateries / (accessed on 22 February 2021).

49. Voo, T.C.; Ballantyne, A.; Ng, C.J.; Cowling, B.J.; Xiao, J.; Phang, K.C.; Kaur, S.; Jenarun, G.; Kumar, V.; Lim, J.M.; et al. Public perception of ethical issues related to COVID-19 control measures in Singapore, Hong Kong, and Malaysia: A crosssectional survey. Preprint 2021. Available online: https://www.medrxiv.org/content/10.1101/2021.03.01.21252710v1 (accessed on 17 July 2021). [CrossRef]

50. Survey Findings on HKSAR Government's Popularity. Available online: http://www.hkiaps.cuhk.edu.hk/eng/survey_result. asp?details=1\&ItemID=Survey000004 (accessed on 7 May 2021).

51. People's Trust in the HKSAR Government. Available online: https:/ /www.pori.hk/pop-poll/government-en/k001.html?lang=en (accessed on 7 May 2021).

52. Weitzman, E.R.; Kelemen, S.; Kaci, L.; Mandl, K.D. Willingness to share personal health record data for care improvement and public health: A survey of experienced personal health record users. BMC Med. Inf. Decis. Mak. 2012, 12, 39. [CrossRef] [PubMed]

53. Ward, P.R. Improving access to, use of, and outcomes from public health programs: The importance of building and maintaining trust with patients/clients. Front. Public Health 2017, 5, 22. [CrossRef] [PubMed]

54. Ienca, M.; Vayena, E. On the responsible use of digital data to tackle the COVID-19 pandemic. Nat. Med. 2020, 26, 463-464. [CrossRef]

55. Struminskaya, B.; Toepoel, V.; Lugtig, P.; Haan, M.; Luiten, A.; Schouten, B. Understanding willingness to share smartphone-sensor data. Public Opin. Q. 2021, 84, 725-759. [CrossRef] [PubMed]

56. Privitera, G.J. Statistics for the Behavioral Sciences, 3rd ed.; SAGE Publications: Thousand Oaks, CA, USA, 2017.

57. South Korea Learned Its Successful Covid-19 Strategy from A Previous Coronavirus Outbreak: MERS. Available online: https:// thebulletin.org/2020/03/south-korea-learned-its-successful-covid-19-strategy-from-a-previous-coronavirus-outbreak-mers / (accessed on 22 February 2021).

58. Park, S.; Choi, G.J.; Ko, H. Information technology-based tracing strategy in response to COVID-19 in South Korea-Privacy controversies. JAMA 2020, 323, 2129-2130. [CrossRef]

59. Who Needs Yellow Fever Vaccination? Available online: https://www.travelhealth.gov.hk/english/faqs/yell_fever.html (accessed on 22 May 2021).

60. What Are the Roadblocks to A "Vaccine Passport”? Available online: https://www.nytimes.com/2021/04/14/travel/covidvaccine-passport-excelsior-pass.html (accessed on 22 May 2021).

61. Lu, J.G.; Jin, P.; English, A.S. Collectivism predicts mask use during COVID-19. Proc. Natl. Acad. Sci. USA 2021, 118, e2021793118. [CrossRef]

62. Cho, Y.N.; Thyroff, A.; Rapert, M.I.; Park, S.Y.; Lee, H.J. To be or not to be green: Exploring individualism and collectivism as antecedents of environmental behavior. J. Bus. Res. 2013, 66, 1052-1059. [CrossRef]

63. Xia, D.; Li, Y.; He, Y.; Zhang, T.; Wang, Y.; Gu, J. Exploring the role of cultural individualism and collectivism on public acceptance of nuclear energy. Energy Policy 2019, 132, 208-215. [CrossRef] 
64. Kim, Y.; Choi, S.M. Antecedents of green purchase behavior: An examination of collectivism, environmental concern, and PCE. Adv. Consum. Res. 2005, 32, 592-599.

65. Yin, J.; Qian, L.; Singhapakdi, A. Sharing sustainability: How values and ethics matter in consumers' adoption of public bicycle-sharing scheme. J. Bus. Ethics 2018, 149, 313-332. [CrossRef]

66. Li, K.K.; Chan, M.W.H.; Lee, S.S.; Kwok, K.O. The mediating roles of social benefits and social influence on the relationships between collectivism, power distance, and influenza vaccination among Hong Kong nurses: A cross-sectional study. Int. J. Nurs. Stud. 2019, 99, 103359. [CrossRef]

67. Coronavirus: Giving Out Patient Details-A Case of Serving Public Good or Invasion of Privacy? Available online: https:/ /www. straitstimes.com/asia/east-asia/coronavirus-giving-out-patient-details-a-case-of-serving-public-good-or-invasion-of (accessed on 22 February 2021).

68. Coronavirus Doxxing Leads to Online Abuse for Young Woman in China. Available online: https://www.scmp.com/news/ china/society/article/3113195/coronavirus-doxxing-leads-online-abuse-young-woman-china (accessed on 22 February 2021).

69. Santos, H.C.; Varnum, M.E.; Grossmann, I. Global increases in individualism. Psychol. Sci. 2017, 28, 1228-1239. [CrossRef] [PubMed]

70. Huang, Z.; Jing, Y.; Feng, Y.U.; Ruolei, G.U.; Zhou, X.; Zhang, J.; Huajian, C.A.I. Increasing individualism and decreasing collectivism? Cultural and psychological change around the globe. Adv. Psychol. Sci. 2018, 26, 2068-2080. [CrossRef]

71. Guillon, M.; Kergall, P. Attitudes and opinions on quarantine and support for a contact-tracing application in France during the COVID-19 outbreak. Public Health 2020, 188, 21-31. [CrossRef] [PubMed]

72. Munzert, S.; Selb, P.; Gohdes, A.; Stoetzer, L.F.; Lowe, W. Tracking and promoting the usage of a COVID-19 contact tracing app. Nat. Hum. Behav. 2021, 5, 247-255. [CrossRef] [PubMed] 